\title{
Experience of Remote Sensing Information Modelling with Grid Computing
}

\author{
Guoyin Cai ${ }^{1}$, Yong Xue ${ }^{1,2 *}$, Jiakui Tang ${ }^{1}$, Jianqin Wang ${ }^{1}$, Yanguang Wang ${ }^{1}$, \\ Ying Luo ${ }^{1}$, Yincui $\mathrm{Hu}^{1}$, Shaobo Zhong ${ }^{1}$, and Xiaosong Sun ${ }^{1}$ \\ ${ }^{1}$ Laboratory of Remote Sensing Information Sciences, Institute of Remote Sensing \\ Applications, Chinese Academy of Sciences, P. Box 9718, Beijing 100101, China \\ ${ }^{2}$ Department of Computing, London Metropolitan University, 166-220 Holloway Road, \\ London N7 8DB, UK \\ \{y.xue@londonmet.ac.uk, cgywj@126.com\}
}

\begin{abstract}
In this paper, we focused on the remote sensing information modeling and determination using Grid computing platform. We have underdone the experiments using remotely sensed images for thermal inertial modeling in Condor system that is one of the Grid Projects existed nowadays worldwide. We divided remote sensing data into several parts and run them on Condor pool and on one single machine. From these tests, the relationship among the work efficiency of image processing in Condor system and the number of the separated parts of image and the number of machines in this system is presented. Given a certain number of machines, a most efficient image size is existed among varies sized images. Besides, the possible causes of the longer put-off in this process are given, and some possible methods to resolve this problem are also presented. It is feasible to use Grid computing system such as Condor to process remote sensing data. And if the postpone problem can be resolved, the work efficiency of Grid systems will be high. Even with so many problems, it is a good thing that Grid systems do many things for you during all of us are in sleep. Our next major task will concentrate on realizing an arithmetic that can read and divide remote sensing images based on image size and number of machines in Grid system automatically, and transfer results back to the submitted machine as a whole data file.
\end{abstract}

\section{Introduction}

Remote Sensing data is characterized by largeness and instantaneousness. Space missions involve the download, from space to ground, of many raw images per day. The analysis and sharing of these huge amounts of data is a big challenge for the remote sensing community. The emerging computational grid technologies are expected to make feasible the creation of a computational environment handling many PetaBytes of distributed data, tens of thousands of heterogeneous computing resources, and thousands of simultaneous users from multiple research institutions (Giovanni et al. 2003).

Grid is an integrated computing and resources environment, or a location for computing resources. Grid can absorb all kinds of resources for computing, and

\footnotetext{
* Corresponding author
} 
transfer them into available, reliable, standard and economic computing ability. Besides computers, computing resources also include web communication abilities, data, instruments and equipments, and even people (Foster and Kesselman 1998). Grid was firstly proposed from using for reference of electric power web. The final goal of the grid is that users can use grid as so convenient as using electric power. Grid can provide final users with common computing abilities that have no relationship with geographic location and computing facilities. Computing abilities provided by Grid cannot be obtained before. The core of Grid is that Grid breaks through various forced constrains on computing resources. It can make people use this computing resources with a new more free and more convenient way to resolve more complicated problems. Finding answers from gird is grid computing, which is a generalized definition. The narrow sense definition of grid computing means organizing distributed computers to resolve complicated science and engineering computing problems cooperatively.

Within the DataGrid project funded by the European Union, an experiment aiming to demonstrate the use of grid technology for remote sensing applications has been carried out. The first results obtained can be found from the paper by Giovanni et al. (2003). Aloisio et al. (2000) presented and discussed an architecture that allows transparent access to remote supercomputing facilities from a web gateway. The implementation exploits the Globus toolkit and provides users with fast, secure and reliable access to parallel applications. They showed the usefulness of their approach in the context of Digital Puglia, an active digital library of remote sensing digital data. The paper by Aloisio and Cafaro (2003) presented an overview of SARA/Digital Puglia (Synthetic Aperture Radar Atlas), a remote sensing environment that shows how grid technologies and high performance computing can be efficiently used to build dynamic earth observation systems for the management of huge quantities of data coming from space missions and for their on-demand processing and delivering to final users. SARA/Digital Puglia is a grid-enabled, high performance digital library of remote sensing images, developed in a joint research project with $\mathrm{CACR} / \mathrm{Caltech}$, ISI/USC and the Italian Space Agency.

Dobashi et al. (2003) proposed an image-based modeling of clouds where realistic clouds were created from satellite images using metaballs. The intention of the paper was for applications to space flight simulators, the visualization of the weather information, and the simulation of surveys of the earth. In the proposed method, the density distribution inside the clouds was defined by a set of metaballs. Parameters of metaballs, such as center positions, radii, and density values, were automatically determined so that a synthesized image of clouds modeled by using metaballs was similar to the original satellite image. They also proposed an animation method for clouds generated by a sequence of satellite images taken at some interval. The usefulness of the proposed method was demonstrated by several examples of clouds generated from satellite images of typhoons passing through Japan.

There are three categories of use of remote sensing data, i.e., processing, analysis, and thematic information modelling and determination. Remote sensing processing involves calibration, transformation, etc. The classification and segmentation etc. from mathematical algorithms are the remote sensing data analysis. It is the thematic information modeling and determination to retrieve in-direct information from remote sensing data via physical models. In this paper, we focused on the remote sensing 
information modeling and determination using Grid computing platform. We have underdone the experiments using remotely sensed images for thermal inertial modeling. First, we will explain the Grid testbed used in the experiments. Following the results, we will discuss the possibilities of use of Grid computing for remote sensing information modelling. Finally, the conclusion and further development will be addressed.

\section{Grid Computing Testbed}

Because of largeness of remote sensing data, it will take a lot of time to process them. It is necessary to have a more efficient processing system to do it. Correspondingly, one of the Grid project-Condor can play a role of sparing time, improving work efficiency (http://www.cs.wisc.edu/condor/overview), which will change our image processing to a large extent, so that the remote sensing can fully exerts its important role of rapid and instantaneous monitoring.

Condor is one of the grid projects in the world. It is the product of the Condor Research Project at the University of Wisconsin-Madison. Compared with the HighPerformance Computing (HPC), Condor provides a High-Throughput Computing (HTC). What the Condor cares is not the number of computed floats in a second, but how many workloads can be done in a period of time. The core of Condor is efficiently using of available resource (http://www.cs.wisc.edu/condor/htc.html). Condor is a specialized workload management system for compute-intensive jobs. Like other full-featured batch systems, Condor provides a job queuing mechanism, scheduling policy, priority scheme, resource monitoring, and resource management. Users submit their serial or parallel jobs to Condor, Condor places them into a queue, chooses when and where to run the jobs based upon a policy, carefully monitors their progress, and ultimately informs the user upon completion (http://www.cs.wisc.edu/ condor/description.html). Condor has two powerful means, one is that it can make available resources more efficient by putting idle machines to work; the other is that it can expand the resources available to users.

Our Grid computing testbed is built on Windows 2000 operating system. It is composed of five Pentium 4 PCs. Three of them have memories of 512MB with a $2.6 \mathrm{GHz}$ frequency of central processing unit (CPU). The other two have memories of $256 \mathrm{MB}$ with a CPU frequency of $2.0 \mathrm{GHz}$. They are connected by local area network (LAN) whose velocity is $100.0 \mathrm{Mbps}$. The installation and configuration of release 6.4.7 of Condor software has been performed on all mentioned PCs by following the instructions reported in http://www.cs.wisc.edu/condor/manual/v6.4. Vanilla environment was adopted in our test. Vanilla is best suitable for Windows platform, but it has no checkpoint and remote system call, two of which are two important exceptional features of Condor. Vanilla environment has no shared file systems, but provide a default file transfer mechanism (see Condor manual at http://www.cs.wisc.edu/condor/manual/v6.4), so lots of files should be transferred to the executable machines before running. The machines in our Condor pool are $\mathrm{Hu}$, Zhong, Wang, Jennyjordan, and Irsa-cgy. $\mathrm{Hu}$ is the Condor Manager and the submitter is Irsa-cgy. Irsa-cgy is also used as the single machine for data processing. 


\section{Remote Sensing Information Modelling}

Our goal is to test the feasibilities of using Grid Computing (Condor system) for Earth surface geophysical parameters determination from remote sensing data, and to examine the work efficiency.

The concept of thermal inertia was introduced to try to take into account both the specific heat and the thermal conductivity in identifying a quantity that would indicate the reluctance of the surface of the Earth (or whatever) to respond to a given heat input. That is, large thermal inertia gives small amplitude oscillations/variations in the surface temperature over a 24-hour period. Let us consider initially the effects of specific heat and thermal conductivity separately. For a given heat input, a large specific heat, $c$, leads to small temperature changes and this is indicative of a large thermal inertia. However, for a given heat input, for a large value of the thermal conductivity (i.e. for a good thermal conductor) more heat is taken away to lower layers and so the surface temperature rise is less. That is, a large thermal conductivity, $\mathrm{K}$, leads to more heat conducted below surface, which in turn leads to a smaller surface temperature rise and this is also indicative of a large thermal inertia.

What is actually done, therefore, is to define thermal inertia

$$
\mathrm{P}=\sqrt{\mathrm{K} \rho \mathrm{c}}
$$

where $\rho=$ density. The $\rho$ is included because what matters here is the specific heat capacity per unit volume whereas $\mathrm{c}$, the specific heat capacity, is usually defined as the heat capacity per unit mass (Cracknell and Xue 1996).

Xue and Cracknell (1995) have developed an operational thermal inertia model. This model will be used in the experiments. The Earth surface albedo and daily maximum surface temperature difference are needed to calculate the thermal inertia values. The Earth surface albedo can be derived from visible band remote sensing data and surface temperature can be determined from thermal band data. Two overpass remote sensing data can be used to calculate the daily maximum surface temperature difference. The Advanced Very High Resolution Radiometer (AVHRR) data of daytime at $14: 30 \mathrm{pm}$ and nighttime at 2:30am were obtained from NOAA (National Oceanic and Atmospheric Administration) satellite. The location of images $\left(84^{\circ} 8^{\prime} 52.63^{\prime \prime} \mathrm{E}-9^{\circ} 9^{\prime} 29.82^{\prime \prime} \mathrm{E}, 38^{\circ} 7^{\prime} 21.37^{\prime \prime} \mathrm{N}-32^{\circ} 1^{\prime} 52.11^{\prime \prime} \mathrm{N}\right)$ is at the border between Qinghai and Xinjiang where a heavy earthquake occurred on November 14, 2001. The image size is $3000 \times 3000$.

\section{Results and Analysis}

Our major tasks of the experiments are testing the working situations of various divisions of images and various sized images running in a single machine and in Condor pool. Firstly, it will mainly for testing the feasibility of using Condor pool to process images and examine how many separated parts of an image has the best work efficiency for Condor pool to run. An image was processed in a single machine whose name is irsa-cgy and the result was recorded. And then the image was divided into 3, 4, 5, and 10 parts in turn. Those separated images were submitted to Condor pool to 
process respectively, each of them was submitted to Condor pool 10 times, and the results were collected. Secondly, based on the above result, the relationship between image size and the work efficiency of Condor pool would be tested and established. Remote sensing images of $1200 \times 720,1200 \times 1000,3000 \times 720,3000 \times 810$ and $3000 \times 1800$ were divided into 3 parts respectively, and then submitted them into Condor pool to run. Each of them was also submitted 10 times.

Table 1. Returned and compiled results from Condor system

\begin{tabular}{|l|c|c|c|}
\hline & Task 1 & Task 2 & Task 3 \\
\hline Postpone time (second) & 16 & 16 & 33 \\
\hline Executing time (second) & 18 & 18 & 14 \\
\hline Total time ( from submitting ) & 34 seconds & 34 seconds & 47 seconds \\
\hline Executable machine & Jennyjordan & wang & zhong \\
\hline
\end{tabular}

Table 1 shows a sample result returned from Condor pool, which records the total information of Condor pool's working including submitted time, executable time, terminal time, machines for executing tasks, and problems occurred during this process. It is feasible to using Condor to process images.

Figure 1 shows the average total time curve and average postpone time curve of tasks running in our Condor pool from 3, 4, 5 and 10 parts of an image with size of $1200 \times 1000$. Our tests show that it has the best work efficiency to separate an image into 3 parts to run in Condor pool. Figure 1 shows that the average postpone time and the average total running time is in a positive proportion, and the postpone time accounts for a lot in the total time.

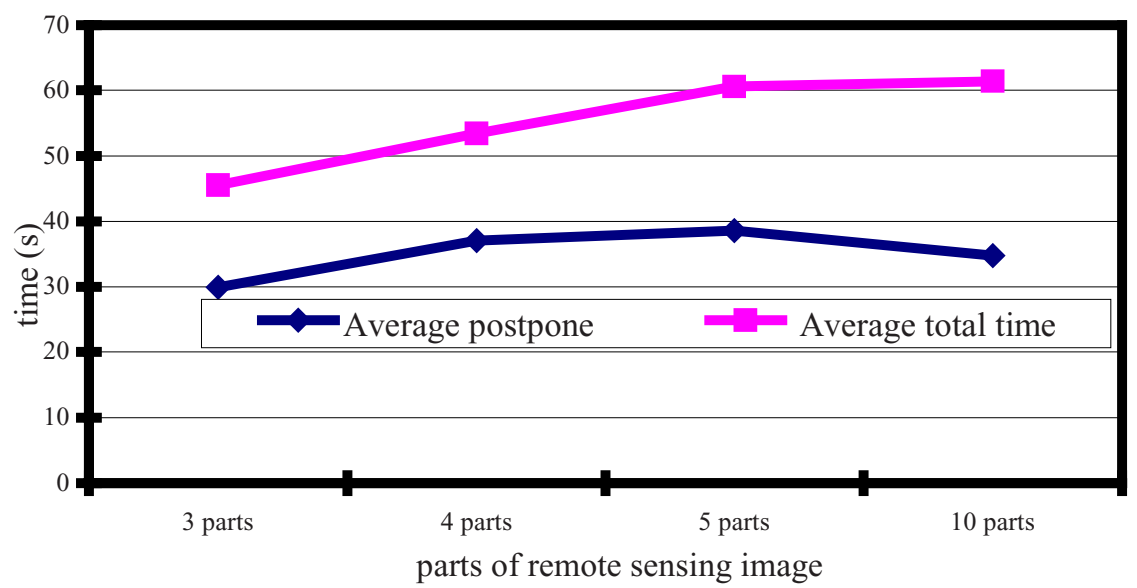

Fig. 1. Average postpone time and average total time curves of the different parts of image with size of $1200 \times 1000$ 


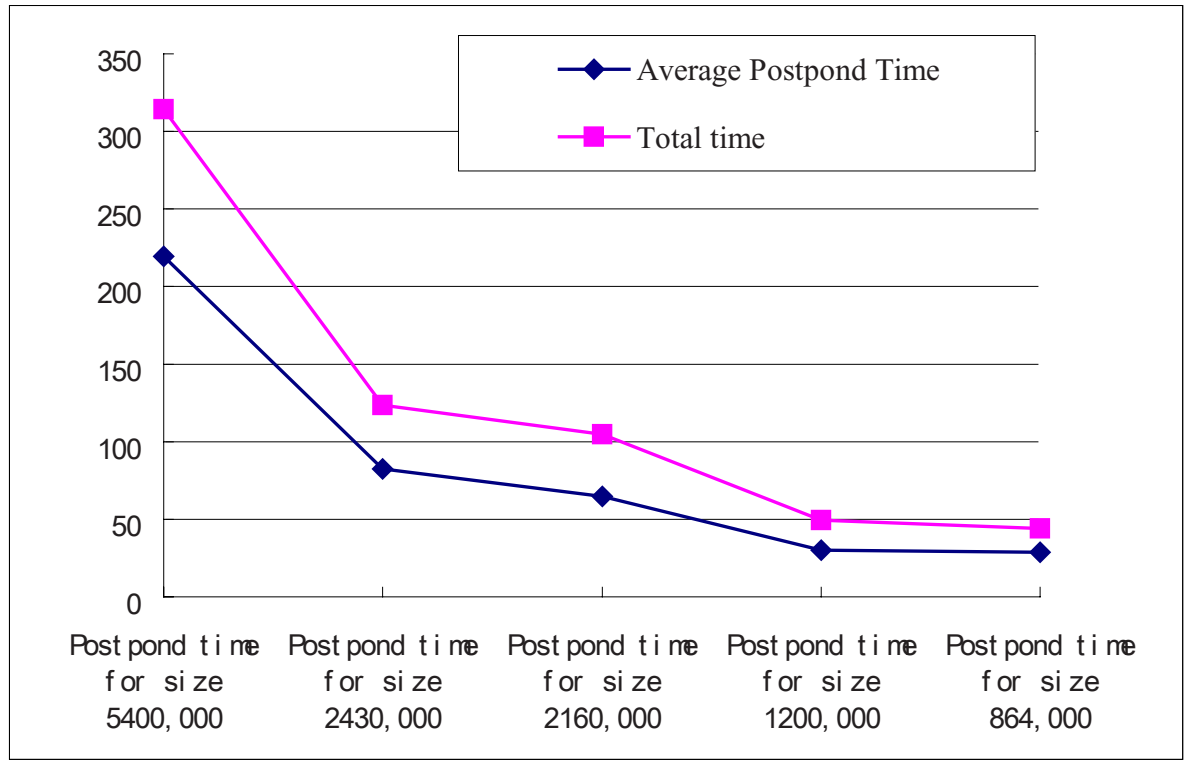

Fig. 2. Returned and compiled results from separated 3 parts of different sized remote sensing images

Table 2. Several parameters to the different sized images

\begin{tabular}{|c|c|c|c|c|}
\hline$\times 10^{4}$ (time unit s) & Time from Condor & Time from S.M & Accelerate index & Postpone/total \\
\hline A.P ${ }^{2}$ for size 540 & 219.4 & & & \\
\hline Total time for 540 & 314.4 & 181 & 0.5757 & 0.69783715 \\
\hline A.P for size 243 & 82.466667 & & & \\
\hline Total time for 243 & 123.5 & 84 & 0.680162 & 0.667746289 \\
\hline A.P for size 216 & 64.733333 & & & \\
\hline Total time for 216 & 104.7 & 73 & 0.69723 & 0.618274435 \\
\hline A.P for size 120 & 30.1 & & & \\
\hline Total time for 120 & 49.4 & 39 & 0.789474 & 0.609311741 \\
\hline A.P for size 86.4 & 28.833333 & & & \\
\hline Total time for 86.4 & 44.1 & 30 & 0.680272 & 0.653817082 \\
\hline
\end{tabular}

${ }^{1}$ : S.M $=$ Single Machine; ${ }^{2}$ : A.P. $=$ Average postpone time 
Figure 2 shows the returned results of different sized image submitted into Condor pool. It indicates that the most suitable size images for Condor pool exists. To our five machines Condor pool, the best efficiency image size is $1200 \times 1000$. But, even the best one, its total running time is longer than the time from a single machine. Table 2 shows the accelerate index which can better indicate system efficiency (Chen and $\mathrm{Li}$ 2003). In our system, accelerate index has not exceed 1, which is a very low efficiency. Table 2 also shows the postpone time accounts for more than 60 percent of total time. One important factor to influent Condor's work efficiency is its long postponing time. Its major possible causes are:

- Transfer files are large and web velocity is low. Generally speaking, remote sensing image data are large, so Condor system needs to transfer many different files to the executable machines before running, and then the executable machines will transfer result files back to the submitted machine. During this process, Condor need to compress files at the submitted machine before transmission, and then recompress files at the executable machine before running, thus, the limited web band will limit file transfer, and then influence work efficiency. Besides, Condor needs to move executable file to the remote executable machines. Which also adds workload to the web band.

- Machine numbers in Condor pool. Relatively, the more the machines are in Condor pool, the less files the Condor system needs to transfer to each executable machine. When task number is less then the machine number, Condor will allocate all of tasks to machines for one time, so it is no necessary to check whether there are available machines and transfer files for second time. Which decreases the postpone time and increases the work efficiency. But it is not the case that the more the machines are, the better the condor system is. Under the circumstance of certain web band, more files that need to move also increase put-off time (Chen and Li 2003).

- It takes time that Condor Manager checks whether the remote machines' disk is enough to accommodate files and Condor system's inner message communications.

Condor focus on the HTC, not the HPC, so it is natural that the postpone time is longer in Condor.

\section{Conclusion and Further Development}

It is feasible to use Grid computing system such as Condor to process remote sensing images. And if the postpone problem can be resolved, the work efficiency of Grid systems will be high. Even with so many problems, it is a good thing that Grid systems do many things for you during all of us are in sleep. Our next major task will concentrate on realizing an arithmetic that can read and divide remote sensing images based on image size and number of machines in Grid system automatically, and transfer results back to the submitted machine as a whole data file.

We can do nothing about web band, but we can increase our machines in Grid system by connecting machines of our two offices together. Then we will do the remote sensing images on Grid system, and the results can be compared with what we have obtained, so that discovery and resolve more problems. 
Acknowledgement. This publication is an output from the research projects "CAS Hundred Talents Program", "Digital Earth" (KZCX2-312) funded by Chinese Academy of Sciences and "Dynamic Monitoring of Beijing Olympic Environment Using Remote Sensing" (2002BA904B07-2) funded by the Ministry of Science and Technology, China.

\section{References}

1. Aloisio G; Cafaro M., 2003, A dynamic earth observation system. PARALLEL COMPUTING 2003, Vol 29, Iss 10, pp 1357-1362.

2. Aloisio, G; Cafaro, M; Falabella, P; Kesselman, C; Williams, R., 2000, Grid computing on the web using the Globus toolkit. Lecture Notes in Computer Science, Vol. 1823, 32-40.

3. Chen Fulong, and Li Ming dong, 2003, Parallel Processing Based on LAN, Journal of Sichuan Teachers College (Natural Sciences), Vol.24, No.1: 59-62.

4. Cracknell, A. P. and Xue, Y., 1996, Thermal Inertia Determination from Space - A Tutorial Review. International Journal of Remote Sensing, 17(3), 431-461.

5. Dobashi, Y., Nishita, T., Yamashita, H., and Okita, T., 2003, Modeling of Clouds from Satellite Images Using Metaballs. www.grid.org

6. Foster, I. and Kesselman, C., (Editors). 1998, The Grid: Blueprint for a new Computing Infrastructure. (Morgan Kaufmann Publishers, Inc.)

7. Giovanni, N. A., Luigi, F. B., and Linford, J., 2003, Grid technology for the storage and processing of remote sensing data: description of an application. In Proceedings of the society of photo-optical instrumentation engineers (SPIE), Vol. 4881, 677-685, 2003.

8. Xue, Y. and Cracknell , A. P., 1995, Advanced thermal inertia modeling. International Journal of Remote Sensing, Vol.16, No.3, 431-446.

9. An Overview of the Condor System, Condor Team, 2003, available from http://www.cs.wisc.edu/condor/overview

10. High-Throughput Computing, Condor Team, 2003, available from http://www.cs.wisc.edu/condor/htc.html

11. Condor Version 6.4.7 Mannual, Condor Team, 2003, available from http://www.cs.wisc.edu/condor/manual/v6.4

12. What is Condor? Condor Team, 2003, available from http://www.cs.wisc.edu/condor/description.html 